\title{
Normalizing Diet in Individuals with Phenylketonuria Treated with Pegvaliase: A Case Series and Patient Perspective
}

\author{
Laurie Bernstein ${ }^{1,2}$ \\ Joyanna Hansen ${ }^{3}$ \\ Christian Kogelmann ${ }^{4}$ \\ Margret Ellerbrok ${ }^{4}$ \\ Maria Gizewska ${ }^{5}$ \\ Sommer Gaughan ${ }^{6}$ \\ Julio Cesar Rocha $\mathbb{1 D}^{7-9}$ \\ Amaya Belanger ${ }^{10}$ \\ Fran Rohr ${ }^{2}$ \\ 'Section of Clinical Genetics and \\ Metabolism, Department of Pediatrics, \\ University of Colorado, Aurora, CO, \\ USA; ${ }^{2}$ Met Ed Co, Boulder, CO, USA; \\ ${ }^{3}$ Department of Molecular and Medical \\ Genetics, Oregon Health \& Science \\ University, Portland, OR, USA; \\ ${ }^{4}$ University Medical Center Hamburg- \\ Eppendorf, Hamburg, Germany; \\ ${ }^{5}$ Department of Pediatrics, \\ Endocrinology, Diabetology, Metabolic \\ Diseases and Cardiology of the \\ Developmental Age, Pomeranian Medical \\ University, Szczecin, Poland; ' Inherited \\ Metabolic Diseases Clinic, Children's \\ Hospital Colorado, Aurora, CO, USA; \\ ${ }^{7}$ Nutrition and Metabolism, NOVA \\ Medical School | Faculdade de Ciências \\ Médicas, Universidade NOVA de Lisboa, \\ Lisboa, I I69-056, Portugal; ${ }^{8} \mathrm{CINTESIS} \mathrm{-}$ \\ Center for Health Technology and \\ Services Research, Porto, Portugal; \\ ${ }^{9}$ Reference Centre of Inherited Metabolic \\ Diseases, Centro Hospitalar \\ Universitário de Lisboa Central, Lisboa, \\ I l69-045, Portugal; ${ }^{10} \mathrm{Hospital}$ \\ Universitario Ramon y Cajal, Madrid, \\ Spain
}

Correspondence: Fran Rohr

Email fran.rohr@met-ed.net

\begin{abstract}
Phenylketonuria (PKU) is one of the most common inherited metabolic disorders, and historically has required affected individuals to follow a severely protein-restricted diet with medical food for life. A novel enzyme substitution therapy, pegvaliase, allows many adults with PKU on therapy to have a reduction in blood phenylalanine (Phe) while on an unrestricted diet. However, there is limited information on optimal nutrition management of individuals with PKU on pegvaliase therapy. This manuscript summarizes a virtual European meeting focused on nutrition management of individuals on pegvaliase therapy, including three case studies and a patient perspective that together provide clinical insights into the real-world management of individuals with PKU on pegvaliase.
\end{abstract}

Keywords: PKU, phenylketonuria, pegvaliase, medical nutrition therapy

\section{Introduction}

Phenylketonuria (PKU) is an autosomal recessive inborn error of metabolism (IEM) caused by pathogenic variants in the phenylalanine hydroxylase (PAH) enzyme. PKU has a global prevalence of approximately 1:23,930 live births and, if untreated, leads to a toxic buildup of phenylalanine that can cause severe intellectual disability. ${ }^{1,2}$ In many countries, PKU is identified via newborn screening (NBS) and newborns are immediately started on medical nutrition therapy, consisting of a Phe-restricted diet supplemented with a Phe-free medical formula (also referred to as a medical food, amino acid mixture or protein substitute). With early treatment, individuals with PKU can have normal cognitive and physical development. ${ }^{3}$

While diet therapy is effective, adherence to the Phe-restricted diet is challenging, particularly for adolescents and adults. ${ }^{4,5}$ The nature of Phe neurotoxicity is very complex and still far from being fully understood. Studies have shown that high brain Phe concentrations may impact synthesis of neurotransmitters and cerebral proteins. ${ }^{6,7}$ High blood Phe may also influence the metabolism of myelin, increase the parameters of oxidative stress, and decrease the activity of enzymes involved in energy production. ${ }^{8}$ Research has further shown that even in early and well-treated adults, there may be persistent neurocognitive issues including depression, anxiety, ADHD, phobias, low self-esteem and social isolation/withdrawal, worsening the quality of life of the patients and their families. ${ }^{9,10}$ Further, many adults discontinue dietary therapy and/or are lost to follow up, highlighting the need for new therapies. ${ }^{11,12}$ 
Pegvaliase (PALYNZIQTM, BioMarin, Novato, CA) is a novel enzyme substitution therapy approved in 2018 by the US Food \& Drug Administration (FDA) for use in adults with blood Phe $>600 \mu \mathrm{mol} / \mathrm{L}$ on current therapy. ${ }^{13}$ It was subsequently approved by the European Medicines Agency (EMA) in 2019 for adolescents and adults aged 16 and over, also with blood Phe $>600 \mu \mathrm{mol} / \mathrm{L} .^{14}$ Pegvaliase is administered by daily subcutaneous injection and degrades Phe via a PAH-independent pathway. ${ }^{15}$ In a randomized discontinuation trial, adults with PKU on pegvaliase showed significant decreases in plasma blood Phe while consuming relatively high amounts of intact protein. ${ }^{15}$ Currently, sapropterin dihydrochloride is the only other approved pharmacologic treatment for PKU, but not all patients are responsive, and a Phe restriction is typically still needed. ${ }^{2}$ Alternatively, individuals with PKU on pegvaliase are usually able to normalize their protein intake and, ideally, consume an unrestricted diet. ${ }^{16}$

Evidence and consensus-based recommendations have been described for the use of pegvaliase in clinical practice, including recommendations for the nutrition management of responders. ${ }^{16,17}$ However, there is limited literature related to the real-world nutrition management of individuals with PKU who have started pegvaliase and normalized their diets after responding to pegvaliase therapy, although some clinical protocols have been published. ${ }^{18,19}$ Furthermore, more information is needed about specific nutritional concerns that may emerge as individuals transition from a Phe-restricted to a higher natural protein diet. Given these gaps, dietitians play a key role in the multidisciplinary care of adult PKU patients, providing nutritional assessment and education, support during pegvaliase induction/titration, and ongoing individualized care. ${ }^{16}$ The recently published European dietitian road map for the nutritional management of phenylketonuria presents a guideline for adequate nutritional status assessment for individuals on pegvaliase therapy, with the primary objective of helping individuals transition from a Phe-restricted diet to a normalized and healthy diet pattern. ${ }^{17}$

The case studies and observations reported in this manuscript are drawn from 3 virtual European programs of metabolic dietitians and physicians: Day 1: Germany, Denmark and the Netherlands; Day 2: Portugal, Italy and Spain; Day 3: Turkey and Greece. While pegvaliase was approved by the EMA, at the time of this meeting only Germany had started patients on pegvaliase. The program included two cases and a patient perspective from U.S.-based clinics, and one case from a clinic in Germany; permission was obtained from each individual case. In addition to presenting the case studies, the broader discussion points from the meetings are summarized herein.

\section{Case Reports}

\section{Case \#I: Patient History}

The first patient case is a 35-year-old female, diagnosed with PKU via NBS, and treated with a Phe-restricted diet and medical formula from infancy. During childhood, she had "fair" adherence to protein-restricted diet (Phe tolerance of about $300-350 \mathrm{mg} /$ day) and excellent medical food intake. As an adult, she was adherent to medical food intake and prescribed Phe intake; despite this, Phe concentrations fluctuated frequently between 120 and 600 $\mu \mathrm{mol} / \mathrm{L}$. She had difficulty with adequate energy intake; of note, this patient had a long history of low body weight, and her BMI typically ranged from low-normal to below normal range. See Table 1 for baseline characteristics and anthropometric data.

At the time of pegvaliase initiation, the patient was taking an amino-acid-based medical formula (PhenylFree ${ }^{\circledR} 2$ and Phenylade ${ }^{\circledR}$ MTE Amino Acid Blend) providing $63 \mathrm{~g}$ Phe-free protein equivalents and $742 \mathrm{kcal}$. She was following a Phe-restricted diet with a goal of about 7 grams protein/350 mg Phe per day, primarily from fruits, vegetables, and grains. Total protein intake was about 70 grams $(1.5 \mathrm{~g} / \mathrm{kg})$. She was not eating any high-quality protein foods, such as meat or dairy. Typically assessed nutrition labs (serum amino acids, prealbumin, and vitamin D) were within the normal range.

This patient previously completed a trial of sapropterin dihydrochloride, but did not experience a decrease in Phe concentrations, so was determined to be a non-responder. The patient met clinic-specific eligibility criteria for pegvaliase as she was an adult patient with PKU, nonresponsive to sapropterin, with a historical blood Phe of greater than or equal to $600 \mu \mathrm{mol} / \mathrm{L}$, although her baseline phe level prior to initiation of pegvaliase was $394 \mu \mathrm{mol} / \mathrm{L}$. She had two healthy pregnancies prior to starting pegvaliase and was not planning any future pregnancies. She was interested in pegvaliase as she was frustrated with her fluctuating blood Phe, as well as the day-to-day struggles of maintaining a strict diet while parenting two young children and working. Her diet goals were to simplify her PKU management by allowing unrestricted intake of whole foods, and to discontinue medical food. Initially, 
Table I Characteristics of Patient Cases

\begin{tabular}{|c|c|c|c|}
\hline & Case \# I & Case \#2 & Case \#3 \\
\hline Age' & 35 & 30 & 34 \\
\hline Sex & $\mathrm{F}$ & $\mathrm{F}$ & $M$ \\
\hline Wt, kg' & 45.2 & 80 & 70.9 \\
\hline BMI' & 16.5 & 32.1 & 27.8 \\
\hline Sapropterin Use ${ }^{\prime}$ & No & No & No \\
\hline Prescribed medical food ${ }^{\prime}$ & $\begin{array}{l}152 \text { g Phenyl-Free }{ }^{\circledR} 2+38 \text { g Phenylade }{ }^{\circledR} \\
\text { MTE AA Blend, } 63 \text { g medial protein }\end{array}$ & $\begin{array}{l}\text { PKU sphere }{ }^{\circledR} \text { (2 packets, } \\
40 \text { g medical protein) }\end{array}$ & $\begin{array}{l}\text { PheBLOC }^{\circledR}, 27 \text { tablets/day, } \\
\text { 14.5 grams medical protein }\end{array}$ \\
\hline Intact protein intake, $g /$ day ${ }^{\prime}$ & 7 & 34 & 110 \\
\hline $\begin{array}{l}\text { Medical food intake at } 12 \text { months on } \\
\text { pegvaliase therapy }\end{array}$ & 0 & 0 & 0 \\
\hline $\begin{array}{l}\text { Intact protein intake at } 12 \text { months on } \\
\text { pegvaliase therapy, grams/day }\end{array}$ & $>80$ & $>100$ & $100-120$ \\
\hline Final pegvaliase dose & $20 \mathrm{mg} /$ day & $10 \mathrm{mg}$ every other day & $10 \mathrm{mg}, 3 \mathrm{x} /$ week \\
\hline
\end{tabular}

Note: 'At pegvaliase initiation.

she was interested in following a vegetarian diet; however, over time her interests shifted to include intake of all foods.

\section{Initiation/Titration}

At pegvaliase initiation, the patient's pre-medications included cetirizine, $\mathrm{H} 2$ antagonists, and ibuprofen (H2 antagonist was discontinued 1 month after initiation of pegvaliase, and is no longer part of standard clinic premedications). At first injection, she experienced side effects of swelling, redness, and soreness at the injection site, which progressed to larger swelling although the severity lessened over time. She also experienced hives in the extremities and trunk; these were treated with cetirizine, diphenhydramine, and a steroid burst $(20 \mathrm{mg}$ prednisone BID for 5 days) and resolved. With ongoing injections, side effects were well managed with cetirizine, and dosing of cetirizine was reduced once the patient was tolerating $20 \mathrm{mg} / \mathrm{d}$ without side effects. The dose titration schedule was adjusted for a slower increase, and overall, titration took about 18 weeks, which is slower than the standard schedule, due to ongoing side effects. The patient's current maintenance dose is $20 \mathrm{mg} /$ day, and she monitors blood Phe/tyrosine monthly (frequency was increased to twice monthly while in titration phase and/ or, while diet changes are occurring).

\section{Diet Modification}

Diet changes were first introduced about 2 months after the pegvaliase dose increased to $20 \mathrm{mg} / \mathrm{day}$, when blood Phe concentrations were $<120 \mu \mathrm{mol} / \mathrm{L}$. She was initially hesitant to introduce higher protein foods due to a life-long history of avoiding these foods. Further, she did not feel comfortable increasing protein by 20 grams, so a 10 -gram increment was introduced. Medical food decreased with each intact protein change, and medical food was discontinued when protein intake met RDA protein needs with Phe concentrations remaining $<120 \mu \mathrm{mol} / \mathrm{L}$ (about 6 months after dose increase to $20 \mathrm{mg} /$ day). Initially, the patient was still consuming modified low-protein foods, so the diet was first transitioned to regular grains and dairy products. Over time, she liberalized her diet to include beans, eggs, nut butters, and eventually chicken and pork. Animal protein was one of the last foods she introduced. She is now on a fully liberalized diet with a goal of at least 80 grams protein per day from both animal and plant-based protein sources. Figure 1 indicates natural protein intake and blood phenylalanine concentrations during diet normalization for this case.

The patient was initially concerned about taking adequate calories, as medical food had provided a large portion of her energy intake. Diet record analysis (MetabolicPro(C) indicated appropriate energy intake, but inadequate intake of vitamin D, vitamin B12, calcium and 


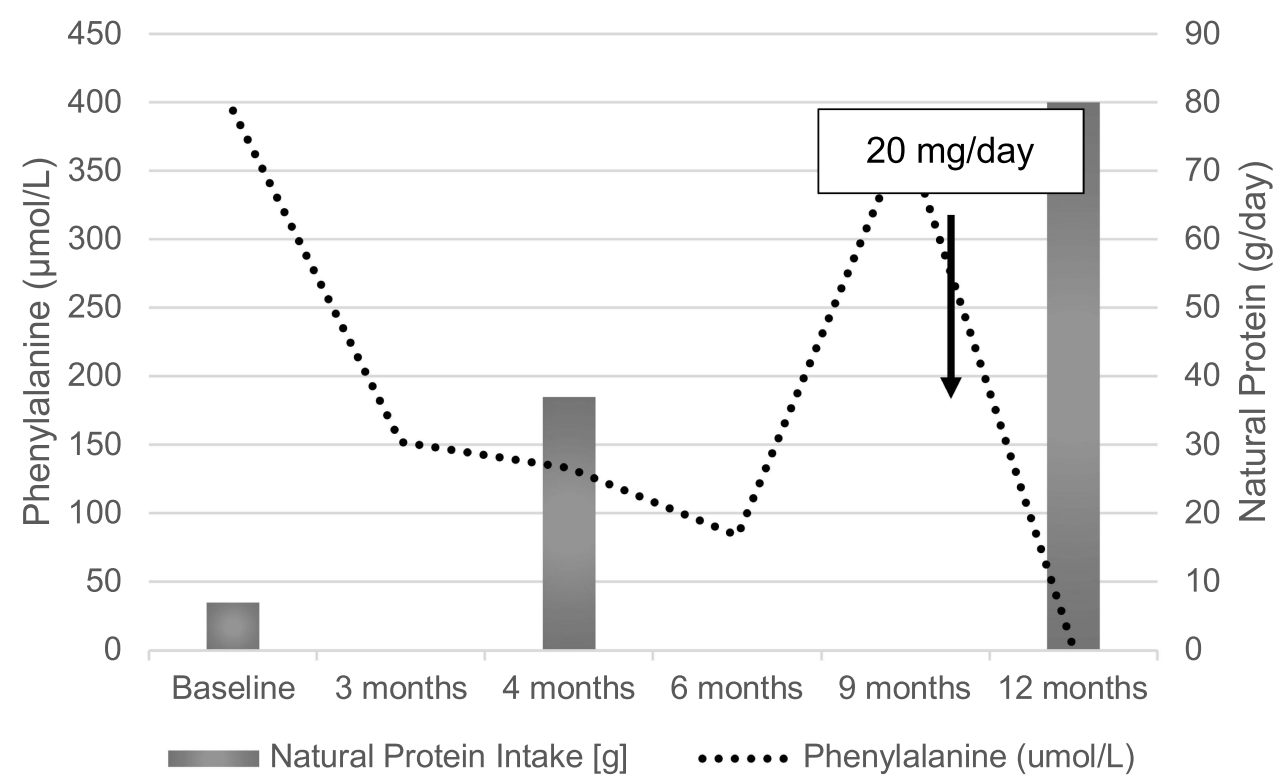

Figure I Blood phenylalanine concentrations and natural protein intake for case $\mathrm{\# I}$.

iron compared to the Dietary Reference Intake (DRI); clinic dietitians counseled the patient to include foods containing these nutrients and start a multivitamin, while patient adjusted to eating a liberalized diet. Overall, the patient is extremely happy with her current diet.

She described the freedom to eat an unrestricted diet as 'life altering' for not only her, but for her family, and reported an improvement in her quality of life. No formal assessment of quality of life was conducted.

She is now able to prepare one meal for her entire family instead of separate meals and is able to choose any restaurant when eating outside the home instead of only those that have lower protein options. Her daily food choices transitioned to those of a more typical American diet with a notable decrease in fruit and vegetable intake and a limited intake of whole grains.

\section{Case \#2: Patient History}

The second case report is a 30 -year-old female, identified via NBS, diagnosed with classical PKU, and treated with a Phe-restricted diet and medical food from infancy (Table 1). Her historical Phe tolerance was $400-600 \mathrm{mg}$ Phe/day, and at this degree of Phe restriction she was able to maintain blood Phe in target range (clinic goal of $<240$ $\mu \mathrm{mol} / \mathrm{L})$. She discontinued the Phe-restricted diet at about 18 years of age, yet as an adult, she did occasionally take some medical food and use special low-protein foods, but mainly followed a vegetarian diet.
The patient never completed a sapropterin dihydrochloride trial as PAH mutations indicated she would not be a responder. She was extremely interested in pegvaliase as she wanted to eat whatever she wished and go to restaurants freely; her primary diet goal was a completely unrestricted diet, as her protein restriction was a burden on her daily life with her wife. She was very motivated to do anything necessary to reach an unrestricted diet, as she was not able to obtain good Phe control and was experiencing self-reported symptoms of depression, sadness, fatigue, and lack of concentration. She was also having severe headaches to the point where she had to quit her job prior to pegvaliase initiation.

At the time of pegvaliase initiation, per diet recall her protein intake was 34 grams of protein $(\sim 1700 \mathrm{mg}$ Phe) per day, but she was not taking any medical food as she thought her protein intake was sufficient. While she ate some higher-protein foods such as cheese, yogurt, and salami, the portion sizes were very small. Her total protein intake was $0.5 \mathrm{~g} / \mathrm{kg}$ protein/day.

At the time of pegvaliase initiation, the clinic asked her to start taking a medical formula on a temporary basis to help her maintain adequate protein intake during the initiation/titration period. At first, the patient declined to restart the medical formula, but after a few weeks she agreed and started a medical formula providing about 40 grams of protein equivalents per day for a total estimated protein intake of 74 grams/day $(0.9 \mathrm{~g} / \mathrm{kg} /$ day $)$. 


\section{Initiation/Titration}

The patient's pre-medication regimen included cetirizine, ibuprofen, and ranitidine; currently, she is only taking the cetirizine (she stopped ibuprofen and ranitidine shortly after initiating pegvaliase). The patient did not experience any side effects after the first injection, but after her second injection she had some arthralgia and swelling. These side effects reoccurred intermittently but were eventually resolved.

\section{Diet Modification}

After increasing to the $10 \mathrm{mg} /$ day dose of pegvaliase, the patient's Phe concentrations dropped quickly. She tried to eat more intact protein, but blood Phe continued to be $<30 \mu \mathrm{mol} /$ L (hypophenylalaninemia), in part because the patient found it difficult to add large amounts of protein initially. When the clinic recognized that she was struggling with increasing protein intake, and her blood Phe remained $<30 \mu \mathrm{mol} / \mathrm{L}$, the clinic requested that she increases blood Phe monitoring from 3 times weekly to 5-7 times weekly. During this period, the clinic tried a $10 \mathrm{mg}$ dose every 2 nd day, and her Phe concentrations increased quickly. The clinic team then changed her dose back to $10 \mathrm{mg}$ daily and her Phe concentrations were more consistent, as in the meantime she had increased her intact protein intake. The patient stopped taking medical formula when she was able to eat $80 \mathrm{~g}$ of intact protein from food. She is currently consuming over 100 grams of protein/ day and loves meat and other high-protein foods. Figure 2 indicates natural protein intake and blood phenylalanine concentrations during diet modifications for this case.

\section{Case \#3: Patient History}

The third case study is a 34-year-old male, who was identified via NBS, diagnosed with classical PKU, and treated with a Phe-restricted diet including medical food from infancy (Table 1). This patient's historical Phe tolerance was about $400 \mathrm{mg} /$ day, or about 8 grams of protein. He followed a Phe-restricted diet during childhood consisting of medical food, fruits, vegetables, and modified low-protein foods, but had difficulty maintaining his diet in his late teens and early adulthood. He had a physically active job, which made it challenging to meet his energy needs without exceeding his Phe tolerance.

At the time of pegvaliase initiation, the patient was consuming a primarily vegetarian diet including regular grains, fruits, and vegetables, as well as limited consumption of higher-protein foods such as eggs and dairy. $\mathrm{He}$ also consumed meat occasionally. At baseline, he was prescribed large neutral amino acid therapy (PheBLOC ${ }^{\text {TM }}$, Nutricia) three times a day with meals (total of 27 tablets/day, providing 14.5 grams Phe-free protein equivalents). He did not track his protein intake, but his Phe concentrations prior to starting pegvaliase were consistently above $1000 \mu \mathrm{mol} / \mathrm{L}$ (goal range of 120-360 $\mu \mathrm{mol} / \mathrm{L}){ }^{2}{ }^{2} \mathrm{~A}$ baseline 24-hour diet recall completed at the time of pegvaliase initiation showed an intact protein of 111 grams/day $(1.6 \mathrm{~g} / \mathrm{kg})$; while he was following a vegetarian diet, he was eating large quantities of dairy and regular grain foods, which contributed to protein intake higher than his protein tolerance.

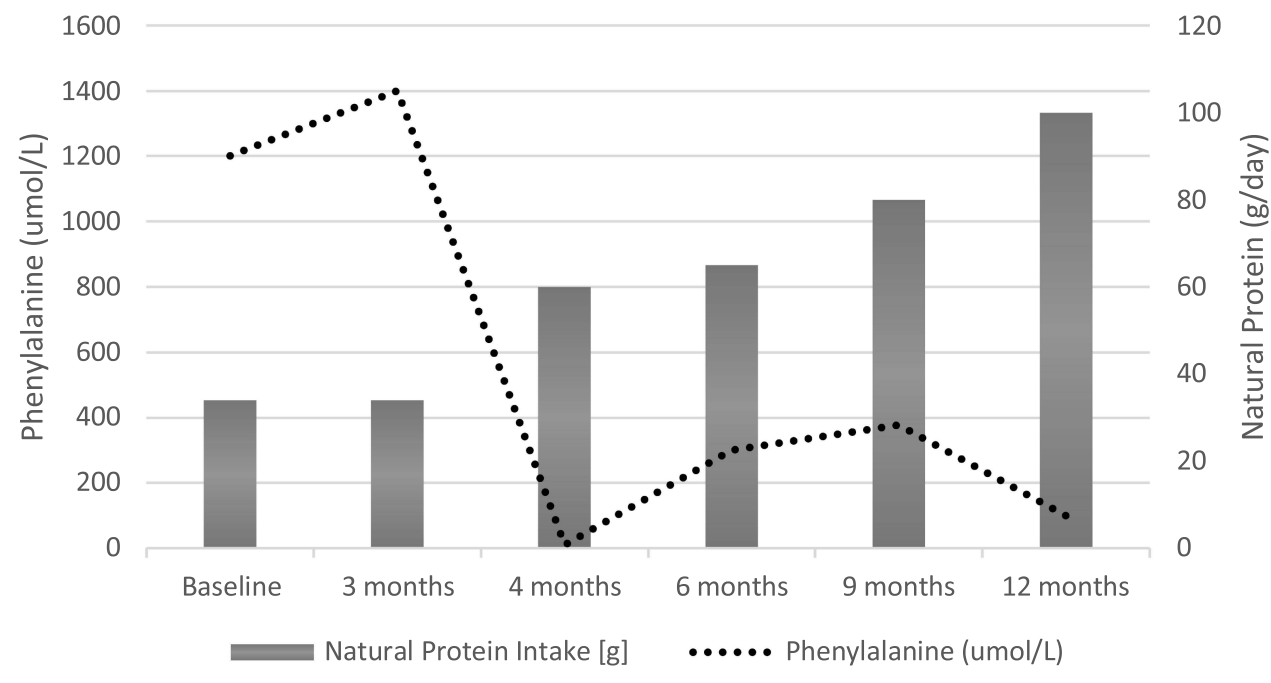

Figure 2 Blood phenylalanine concentrations and natural protein intake for case \# 2 . 
He was interested in starting pegvaliase for a variety of reasons, including his relatively low protein tolerance and high energy needs, and self-reported difficulty with concentration and mood. Our patient's diet goals after reaching efficacy on pegvaliase were to normalize his diet without the need for large neutral amino acid therapy. He had never completed a sapropterin trial.

\section{Initiation/Titration}

When starting pegvaliase, he followed the standard induction/titration schedule with the following pre-medications: $\mathrm{H} 1$ antagonist (loratadine), $\mathrm{H} 2$ antagonist (ranitidine), and naproxen (as needed). During the induction/titration period, he did experience intermittent minor site injection reactions, which resolved, and occasional hives localized to the injection site, which resolved with loratadine. The patient discontinued ranitidine after about 6 months on pegvaliase.

\section{Diet Modification}

After approximately 2 months on therapy, the patient titrated up to $20 \mathrm{mg}$ of pegvaliase once per day. About 1 month after starting the $20 \mathrm{mg} /$ day dose, his Phe concentrations decreased rapidly to $<30 \mu \mathrm{mol} / \mathrm{L}$. Clinic dietitians worked with patient to increase protein gradually in 10-20 gram increments, but blood Phe remained less than 30 $\mu \mathrm{mol} / \mathrm{L}$. Given low Phe concentrations, the patient quickly transitioned to eating an unrestricted diet, and discontinued the PheBLOC ${ }^{\circledR}$. He added in many high-protein foods, including chicken, fish, pork, eggs, and even whey protein supplements, never having difficulty adding these highprotein foods as he was excited to be able to eat an unrestricted diet. Despite a high intact protein intake, our patient's Phe concentrations remained $<30 \mu \mathrm{mol} / \mathrm{L}$. He experienced migraines that he attributed to very low Phe concentrations. Given that the patient was already on a completely unrestricted diet, his pegvaliase dose was titrated down from 20 to $10 \mathrm{mg}$ daily. Blood Phe increased slightly but remained below goal range. The pegvaliase dose was titrated further down to $10 \mathrm{mg} 3 \mathrm{x} / \mathrm{week}$, and blood Phe was in the target range. The clinic team reduced the pegvaliase dose to $10 \mathrm{mg} \mathrm{2x} /$ week, but this resulted in an increase in blood Phe, so the dose was adjusted back to $10 \mathrm{mg} \mathrm{3x} /$ week. The patient remains at this dose and is able to maintain Phe concentrations between above 30 $\mu \mathrm{mol} / \mathrm{L}$ and less than $360 \mu \mathrm{mol} / \mathrm{L}$ while eating a completely unrestricted diet and without the need for large neutral amino acid therapy. Figure 3 indicates natural protein intake and blood phenylalanine concentrations during diet normalization for this case.

\section{Patient Perspective}

A 17-year-old female with PKU from the US who started pegvaliase at age 16 years and her mother described the family's experience with the induction and titration phase of her trial. The patient had moderate PKU (highest Phe

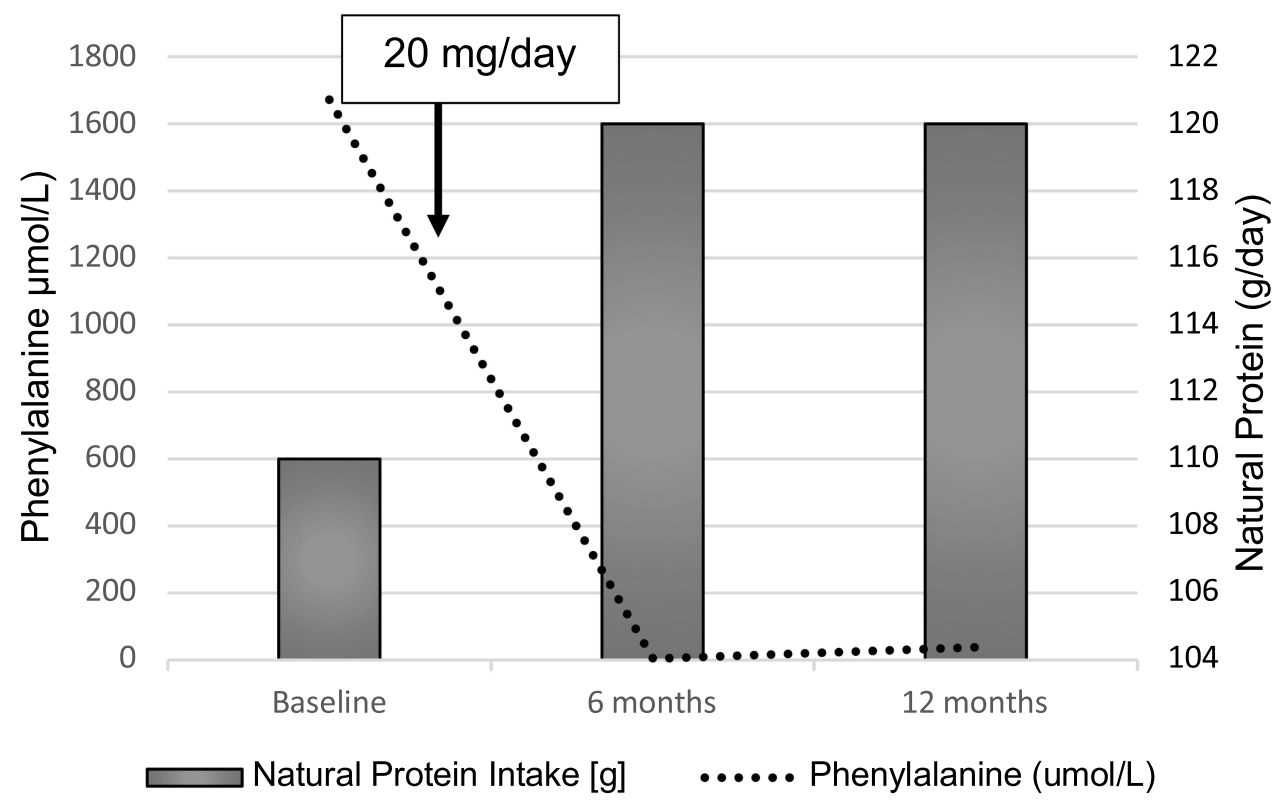

Figure 3 Blood phenylalanine concentrations and natural protein intake for case \#3. 
concentration prior to initiation of a Phe-restricted diet was $714 \mu \mathrm{mol} / \mathrm{L}$ ) and was treated with a Phe-restricted diet (approximately $350 \mathrm{mg}$ Phe or $7 \mathrm{~g}$ protein/d on Simplified $\operatorname{Diet}^{20}$ ) and medical food providing $60 \mathrm{~g}$ protein, as well as sapropterin. Since pegvaliase is only approved for adults in the US, off-label insurance coverage for pegvaliase was sought because her metabolic physician and dietitian, along with the family, felt it was important for the patient to start the drug at an earlier age because of the need for better blood Phe control, the benefit of having family support during high school versus while at college at age 18 , and the patient's history of obesity and the potential benefit of being able to improve her diet.

The patient followed a standard pegvaliase dose titration schedule, reaching a dose of $20 \mathrm{mg} / \mathrm{d}$ by 12 weeks after drug initiation. She experienced mild injection site reactions and mild-to-moderate gastrointestinal distress (pain, nausea) starting at about 3 weeks and continuing throughout the induction and titration phase; this was significant enough, at times, to prevent her from attending school. Baseline blood Phe concentration was $589 \mu \mathrm{mol} / \mathrm{L}$ (average of three filter paper specimens). Blood Phe appeared to be decreased at 12 weeks but then fluctuated between $400 \mu \mathrm{mol} / \mathrm{L}$ and $648 \mu \mathrm{mol} / \mathrm{L}$ over the time she was on $10 \mathrm{mg} / \mathrm{d}$. At week 23, the dose was increased to $20 \mathrm{mg} / \mathrm{d}$ and her blood Phe decreased within 10 days weeks to $0 \mu \mathrm{mol} / \mathrm{L}$ (last data available at the time of the meeting). She remained on sapropterin throughout the pegvaliase induction and titration and discontinued it as soon as she responded.

The patient and mother described the difficulty in maintaining the Phe-restricted diet during a long titration period, especially when it appeared that the blood Phe concentration was decreasing. By the time the patient responded at a dose of $20 \mathrm{mg} / \mathrm{d}$, she had (on her own) stopped taking medical food and was consuming approximately $30 \mathrm{~g}$ of intact protein daily. Once she responded, her metabolic team first recommended discontinuing sapropterin and then increasing the intact protein to $60 \mathrm{~g} /$ $\mathrm{d}$ because the patient had already included several highprotein options (meat and dairy) in her diet. At the time of the virtual meeting, the patient had been on a normal diet for 2 weeks. Her mother felt that her daughter would benefit from education about what constitutes healthy eating (which foods, serving sizes) because she has had no experience with normal eating, and because, as an adolescent, she may be more open to hearing messages about good health from a professional versus from her parents.
The patient and her mother also commented that although they were aware that there was no information about pegvaliase in pregnancy, this was not an immediate concern and was not a deterrent to choosing pegvaliase therapy.

\section{Meeting Discussion}

Additional presentations and discussions during the virtual European meeting highlighted anticipatory considerations related to patient selection and clinic readiness.

\section{Clinic Preparation}

Proper preparation of the clinical team to start pegvaliase was discussed in the virtual meeting. At a minimum, questions that should be addressed prior to starting pegvaliase include: the process for obtaining insurance authorization; logistics of providing pegvaliase to patients; emergency clinic contact for reporting side effects; assessment of clinic capacity; and development of patient education materials including patient expectations.

\section{Patient Selection}

While ideally all individuals with PKU who qualify per the pegvaliase label requirements would be offered the drug, due to insurance authorization requirements and the significant clinician time involved in initiating pegvaliase, not all patients may be able to start pegvaliase right away. There was no consensus about who should be offered pegvaliase first, rather that this is a matter of clinical judgement and available resources. There was agreement that individuals with PKU who are well controlled on a PKU diet should be equally eligible for pegvaliase as those with poor metabolic control. Pegvaliase in individuals with PKU ages 16-18 is not approved in the US, yet is approved by the EMA; however, in some cases, as noted in the patient perspective, off-label insurance coverage may be granted if there is justification.

Pegvaliase is currently not recommended for women who are pregnant or planning to become pregnant in the near future. ${ }^{16}$ Of the two females reported in the case studies, neither intended to become pregnant, nor was pregnancy a current concern for the patient who offered her perspective. The safety of pegvaliase during pregnancy has not been established and is a question asked by many patients and providers. Notably, one recent case study has been published detailing the successful use of pegvaliase during pregnancy. ${ }^{21}$ 


\section{Discussion}

The 3-day virtual meeting provided insight into the experiences and expectations of clinicians from the US and Europe who are or will be working with individuals with PKU on pegvaliase therapy. Published guidance from US and European experts outlines considerations for achieving the best outcomes, recognizing that real-world experience is limited and treatment is individualized. ${ }^{16,17}$ Dietitians from the US and Europe shared similar experiences regarding the nutrition concerns and challenges when transitioning a patient from a Phe-restricted diet to an unrestricted diet in patients who respond to pegvaliase therapy.

\section{Diet Normalization and Nutritional Considerations}

Dietitians continue to play an important role in the management of individuals with PKU starting pegvaliase, particularly during the induction and titration phases of therapy, but also during later stages. ${ }^{17}$ In addition to counseling patients about when and how to begin diet liberalization, metabolic dietitians can help individuals with PKU choose high-quality protein sources and develop a healthy, higher-protein diet pattern. When normalizing the diet, education should focus on incorporating "healthy" proteins, not just increasing protein overall, but also a healthy diet for prevention of comorbidities. ${ }^{17}$

Two of the case studies (Case 1 and 2) highlighted the psychological impact of transitioning from a proteinrestricted to a liberalized diet. Development and implementation of food neophobia questionnaires or other tools may provide guidance for dietitians working with individuals with PKU starting pegvaliase, as food neophobia may play a role in the willingness of individuals on pegvaliase therapy to try new foods. ${ }^{22}$

Two of the case studies (Cases 2 and 3) highlighted individuals who experienced hypophenylalaninemia (blood Phe $<30 \mu \mathrm{mol} / \mathrm{L}$ ) at either the $10 \mathrm{mg}$ or $20 \mathrm{mg}$ pegvaliase dose and were able to decrease the frequency of dosing to less than once per day while eating a normal diet and maintaining blood Phe $<360 \mu \mathrm{mol} / \mathrm{L}$. This corresponds to the 2018 guidelines that recommend decreasing pegvaliase dosage if the addition of intact protein does not adequately increase blood Phe concentrations in patients with hypophenylalaninemia. ${ }^{16}$ Notably, these individuals had an excellent response to pegvaliase and may not be reflective of the broader population of individuals with PKU on pegvaliase therapy.
The three case study individuals were all able to normalize their diet and discontinue medical food while maintaining Phe concentrations $<360 \mu \mathrm{mol} / \mathrm{L}$. However, the transition from a Phe-restricted diet to a normal diet was not without challenges, including the need for nutrition education and monitoring. Ideally, individuals will be able to maintain positive aspects of the Phe-restricted diets, such as an emphasis on fruit and vegetable intake, while meeting protein needs from intact protein rather than medical formula.

Further experience and discussion are needed regarding the appropriate Phe threshold for starting to increase dietary protein for individuals with PKU on a proteinrestricted diet at the time of pegvaliase initiation. In some cases, blood Phe concentrations may decrease very quickly once the patient starts to respond, and the metabolic team may need to quickly work with the individual to increase intact protein intake.

\section{Conclusions}

In conclusion, these case reports detail the similar clinical experience of metabolic dietitians in the US and Germany working with individuals with PKU on pegvaliase therapy, emphasizing that drug titration and diet normalization must be individualized for each patient. With ongoing research and clinical experience, future nutrition management and identification of educational needs of individuals with PKU on pegvaliase therapy will continue to evolve.

\section{Synopsis}

Metabolic dietitians are an important part of the multidiscplinary care team for individuals with PKU on pegvaliase therapy, particularly as individuals transition away from a protein-restricted diet.

\section{Informed Consent Statement}

Written informed consent has been obtained from the patients' profile in these case reports to publish this paper.

\section{Author Contributions}

All authors made a significant contribution to the work reported, whether that is in the conception, study design, execution, acquisition of data, analysis and interpretation, or in all these areas; took part in drafting, revising or critically reviewing the article; gave final approval of the version to be published; have agreed on the journal to which the article has been submitted; and agree to be accountable for all aspects of the work. 


\section{Funding}

The virtual meeting and the manuscript preparation was supported by a BioMarin educational grant (Grant \#2020GR-005030). The manuscript was developed independently of BioMarin.

\section{Disclosure}

J.H. is an employee of BioMarin Pharmaceutical Inc. as of August 9, 2021. J.H. has received a research grant from Vitaflo and has received honoraria as a consultant and/or speaker for Aeglea, BioMarin, Nutricia, Met Ed, and Vitaflo. J.C.R. received research grants from BioMarin, Glutamine and Cambrooke, as well as personal/lectures fees from BioMarin, Applied Pharma Research, Nutricia, Merck Serono, Vitaflo, Cambrooke, PIAM and Lifediet. M.G. has been a member of the Scientific Advisory Board of Merck-Serono SA, and BioMarin and received honoraria as a consultant and/or speaker for Merck Serono SA, BioMarin, Nutricia. Sanofi-Aventis, Swixx Biopharma and Vitaflo. C.K. and M.E. have received honoraria as a speaker for BioMarin. C.K. has been a speaker for Vitaflo and Nutricia; also reports personal fees from Orphan and VDD Germany. A.B. has been a member of Scientific Advisory Boards of Merck-Serono SA, BioMarin, Nutricia and has received honoraria from Merck-Serono SA, BioMarin, Nutricia/Danone. F.R. and L.B. are managing partners of Met Ed that received grant support from BioMarin. The authors report no other conflicts of interest in this work.

\section{References}

1. Hillert A, Anikster Y, Belanger-Quintana A, et al. The Genetic Landscape and Epidemiology of Phenylketonuria. Am J Hum Genet. 2020;107(2):234-250. doi:10.1016/j.ajhg.2020.06.006

2. Vockley J, Andersson HC, Antshel KM, et al. Phenylalanine hydroxylase deficiency: diagnosis and management guideline. Genet Med 2014;16(2):188-200. doi:10.1038/gim.2013.157

3. Singh RH, Rohr F, Frazier D, et al. Recommendations for the nutrition management of phenylalanine hydroxylase deficiency. Genet Med. 2014;16(2):121-131. doi:10.1038/gim.2013.179

4. Brown CS, Lichter-Konecki U. Phenylketonuria (PKU): a problem solved? Mol Genet Metab Rep. 2016;6:8-12. doi:10.1016/j. ymgmr.2015.12.004

5. Cazzorla C, Bensi G, Biasucci G, et al. Living with phenylketonuria in adulthood: the PKU ATTITUDE study. Mol Genet Metab Rep. 2018;16:39-45. doi:10.1016/j.ymgmr.2018.06.007

6. Hoeksma M, Reijngoud DJ, Pruim J, de Valk HW, Paans AM, van Spronsen FJ. Phenylketonuria: high plasma phenylalanine decreases cerebral protein synthesis. Mol Genet Metab. 2009;96(4):177-182. doi:10.1016/j.ymgme.2008.12.019
7. Pilotto A, Blau N, Leks E, et al. Cerebrospinal fluid biogenic amines depletion and brain atrophy in adult patients with phenylketonuria. $J$ Inherit Metab Dis. 2019;42(3):398-406. doi:10.1002/jimd.12049

8. Dobrowolski SF, Phua YL, Sudano C, et al. Phenylalanine hydroxylase deficient phenylketonuria comparative metabolomics identifies energy pathway disruption and oxidative stress. Mol Genet Metab. 2021. doi:10.1016/j.ymgme.2021.04.002

9. Ashe K, Kelso W, Farrand S, et al. Psychiatric and Cognitive Aspects of Phenylketonuria: the Limitations of Diet and Promise of New Treatments. Front Psychiatry. 2019;10:561. doi:10.3389/ fpsyt.2019.00561

10. Bilder DA, Kobori JA, Cohen-Pfeffer JL, Johnson EM, Jurecki ER, Grant ML. Neuropsychiatric comorbidities in adults with phenylketonuria: a retrospective cohort study. Mol Genet Metab. 2017;121 (1):1-8. doi:10.1016/j.ymgme.2017.03.002

11. Burgess NM, Kelso W, Malpas CB, et al. The effect of improved dietary control on cognitive and psychiatric functioning in adults with phenylketonuria: the ReDAPT study. Orphanet J Rare Dis. 2021;16 (1):35. doi:10.1186/s13023-020-01668-2

12. Burton BK, Leviton L. Reaching out to the lost generation of adults with early-treated phenylketonuria (PKU). Mol Genet Metab. 2010;101(2-3):146-148. doi:10.1016/j.ymgme.2010.06.006

13. US Food and Drug Administration. Palynziq Full Prescribing Information. Available from: https://www.accessdata.fda.gov/drug satfda_docs/label/2018/761079s000lbl.pdf. Accessed December 20, 2021

14. European Medicines Agency. Palynziq. Available from: https://www.ema. europa.eu/en/medicines/human/EPAR/palynziq. Accessed December 20, 2021.

15. Thomas J, Levy H, Amato S, et al. Pegvaliase for the treatment of phenylketonuria: results of a long-term Phase 3 clinical trial program (PRISM). Mol Genet Metab. 2018;124(1):27-38. doi:10.1016/j. ymgme.2018.03.006

16. Longo N, Dimmock D, Levy H, et al. Evidence- and consensus-based recommendations for the use of pegvaliase in adults with phenylketonuria. Genet Med. 2019;21(8):1851-1867. doi:10.1038/ s41436-018-0403-z

17. Rocha J, Bausell H, Bélanger-Quintana A, et al. Development of a practical dietitian road map for the nutritional management of phenylketonuria (PKU) patients on pegvaliase. Mol Genet Metab Rep. 2021;28:100771. doi:10.1016/j.ymgmr.2021.100771

18. Sacharow S, Papaleo C, Almeida K, et al. First 1.5 years of pegvaliase clinic: experiences and outcomes. Mol Genet Metab Rep. 2020;24:100603. doi:10.1016/j.ymgmr.2020.100603

19. Lah M, McPheron M. Palynziq clinic: one year and 43 patients later. Mol Genet Metab. 2021;133(3):250-256. doi:10.1016/j. ymgme.2021.05.006

20. Bernstein L, Burns C, Sailer-Hammons M, Kurtz A, Rohr F. Multiclinic Observations on the Simplified Diet in PKU. $J$ Nutr Metab. 2017;2017:4083293. doi:10.1155/2017/4083293

21. Boyer M, Skaar J, Sowa M, Tureson JR, Chapel-Crespo CC, Chang R. Continuation of pegvaliase treatment during pregnancy: a case report. Mol Genet Metab Rep. 2021;26:100713. doi:10.1016/ j.ymgmr.2021.100713

22. Evans S, Daly A, Chahal S, MacDonald J, MacDonald A. Food acceptance and neophobia in children with phenylketonuria: a prospective controlled study. J Hum Nutr Diet. 2016;29 (4):427-433. doi:10.1111/jhn. 12346 


\section{Publish your work in this journal}

Nutrition and Dietary Supplements is an international, peerreviewed, open access journal focusing on research into nutritional requirements in health and disease, impact on metabolism and the identification and optimal use of dietary strategies and supplements necessary for normal growth and development. The journal welcomes submitted papers covering original research, basic science, clinical \& epidemiological studies, reviews and evaluations, guidelines, expert opinion and commentary, case reports and extended reports. The manuscript management system is completely online and includes a very quick and fair peer-review system, which is all easy to use. Visit http://www.dovepress.com/testimonials.php to read real quotes from published authors. 